\title{
Dupilumab reduces opacification across all sinuses and related symptoms in patients with CRSwNP*
}

\section{Claus Bachert ${ }^{1}$, S. James Zinreich², Peter W. Hellings ${ }^{3}$, Joaquim Mullol', Daniel L. Hamilos ${ }^{5}$, Philippe Gevaert ${ }^{1}$, Robert M. Naclerio ${ }^{6}$, Nikhil Amin”, Vijay N. Joish ${ }^{8}$, Chunpeng Fan', Donghui Zhang ${ }^{9}$, Heribert Staudinger ${ }^{9}$, Gianluca Pirozzi ${ }^{9}$, Neil M.H. Graham, Asif Khan ${ }^{10}$, Leda P. Mannent ${ }^{10}$}

Rhinology 58: 1, 10 - 17, 2020

https://doi.org/10.4193/Rhin18.282

*Received for publication:

November 23, 2018

Accepted: July 29, 2019

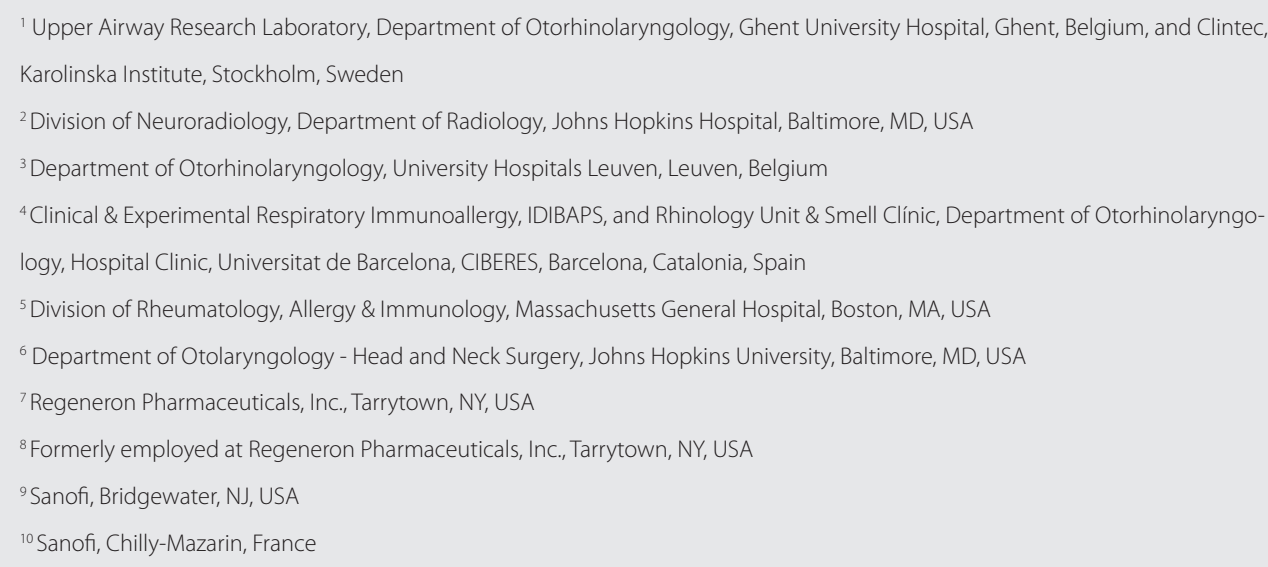

\section{Abstract}

Background: Chronic rhinosinusitis with nasal polyposis (CRSwNP) is associated with substantial sinus opacification. In a phase 2a study (NCT01920893), dupilumab, a fully human anti-IL-4Ra monoclonal antibody, improved outcomes in CRSwNP refractory to intranasal corticosteroids. We evaluated dupilumab's effect on sinus opacification in relation to effects on nasal polyp burden, symptoms, and health-related quality of life (HRQoL) in patients with CRSwNP.

Methodology: 16-week randomized, double-blind, placebo-controlled, parallel-group study in 60 adults with CRSwNP. Patients received weekly subcutaneous dupilumab 300-mg or placebo and daily mometasone furoate nasal spray. Sinus opacification was assessed using standard and Zinreich-modified Lund-Mackay (zLMK) scoring. Correlation was assessed between zLMK score and CRSwNP endpoints, including nasal polyp score (NPS), SNOT-22, daily symptom scores, and UPSIT smell-test score.

Results: Baseline characteristics were similar across treatment groups. Mean \pm SD baseline LMK scores of 18.7 \pm 5.5 (placebo) and $18.6 \pm 5.0$ (dupilumab) indicated severe disease with extensive opacification involving all sinuses. Baseline LMK and LMK scores correlated with NPS severity and loss of sense of smell (daily symptoms; SNOT-22 smell/taste; loss of sense of smell [UPSIT]). At Week 16, dupilumab-treated patients had significantly improved sinus opacification measured by LMK in all individual sinuses vs placebo. Dupilumab also showed similar efficacy with zLMK, with only small differences from LMK, and correlated with SNOT22 smell/taste. The most common adverse events were nasopharyngitis, injection-site reactions, and headache.

Conclusions: In patients with CRSwNP, baseline LMK showed extensive sinus opacification and correlated with symptoms, HRQoL, and hyposmia. Dupilumab treatment reduces opacification across all sinuses and related symptoms in patients with CRSwNP.

Key words: nasal polyps, inflammation, sinusitis, rhinitis, therapeutics 


\section{Introduction}

Chronic rhinosinusitis (CRS) is an inflammatory condition of the sinuses, with prevalence estimated as high as $12 \%$ in Western populations ${ }^{(1)}$. It is characterized by nasal congestion, decreased or lost sense of smell, nasal discharge (anterior/posterior), facial pain and pressure, and headache, often lasting for many years. Based on endoscopic findings, the condition can be divided into CRS with or without nasal polyposis (CRSwNP and CRSsNP, respectively).

CRSwNP patients experience significant nasal and paranasal sinus inflammation, loss of sense of smell, high disease burden, and significant impact on their health-related quality of life (HRQoL) ${ }^{(2,3)}$.

In describing mucosal drainage from the sinuses, Messerklinger (4) outlines the mucocilliary passages from the different sinuses and their opening into the middle and superior meatus and their drainage into the nasopharynx. Mucosal drainage of the frontal, maxillary, and anterior ethmoid sinuses is therefore accomplished by the anterior ostiomeatal channels and drainage of the posterior ethmoid and sphenoid sinuses is carried out by the posterior ostiomeatal channels.

Recent studies providing a more accurate description of the inflammatory mechanisms involved in CRS ${ }^{(5)}$ and correlating these mechanisms with different phenotypes support the concept of treatments targeted against specific mediators of inflammation in CRSwNP. Type 2 inflammation characterized by the release of signature cytokines interleukin (IL) 4 (IL-4), IL-13, and IL-5 via both the innate and adaptive immune pathways plays a central role in the disease pathobiology of the vast majority of Western patients with CRSwNP.

Dupilumab, a fully human Veloclmmune ${ }^{\circledR}$-derived ${ }^{(6,7)}$ monoclonal antibody directed against IL-4 receptor $\alpha$, inhibits signaling of IL-4 and IL-13, cytokines that are key drivers of type 2 immune diseases such as CRSwNP, atopic dermatitis (AD), asthma, allergic rhinitis, eosinophilic esophagitis, and food allergies, which often present as overlapping comorbidities.

Dupilumab is approved by the US Food and Drug Administration ${ }^{(8)}$ as an add-on maintenance treatment in patients with moderate-to-severe asthma aged $\geq 12$ years with an eosinophilic phenotype or with oral corticosteroid-dependent asthma (9-11), in Japan, for patients aged $\geq 12$ years with severe or refractory bronchial asthma whose symptoms are inadequately controlled with existing therapies ${ }^{(12)}$, and by the European Medicines Agency ${ }^{(13)}$, as an add-on maintenance treatment in patients aged $\geq 12$ years with severe asthma with type 2 inflammation characterized by increased blood eosinophils and/or raised fractional exhaled nitric oxide who are inadequately controlled with high dose inhaled corticosteroids plus another medicinal product for maintenance treatment. Dupilumab is also approved for the treatment of patients with inadequately controlled, moderate-to-severe $A D$, aged $\geq 12$ years in the USA ${ }^{(8)}$, and adults in the EU ${ }^{(13)}$ and other countries ${ }^{(14-16)}$, and has demonstrated positive results in a proof-of-concept study for patients with eosinophilic esophagitis ${ }^{(17)}$.

In a proof-of-concept study (NCT01920893), dupilumab, in conjunction with daily mometasone furoate nasal spray (MFNS) therapy, was shown to improve endoscopic, clinical, and patientreported outcomes in patients with CRSwNP refractory to intranasal corticosteroids ${ }^{(18)}$.

In the abovementioned dupilumab proof-of-concept study, opacification assessed by computed tomography (CT) scan was reported as total Lund-Mackay (LMK) CT score only. Given the extensive disease involving all sinuses in this cohort, we assessed individual sinus-specific LMK scores to comprehensively evaluate the effect of dupilumab treatment across individual areas of sinus opacification. We also assessed opacification using the Zinreich-modified Lund-Mackay (zLMK) CT scoring system, a more granular adaptation of LMK ${ }^{(19)}$ that includes segmentation of the anterior and posterior ostiomeatal channels (OMC) ${ }^{(20)}$. Furthermore, to assess whether the improvement in sinus opacification parallels previously reported dupilumab effects on nasal polyp burden (nasal polyp score [NPS]), symptom severity, and HRQoL disease burden in patients with CRSwNP, the treatment effect of dupilumab on these outcome measures is placed in context relative to the sinus $\mathrm{CT}$ score changes through correlation analysis.

\section{Materials and methods}

Study design and population

We reported the primary study design and population in detail elsewhere ${ }^{(18)}$ (Appendix Figure S1). Briefly, we conducted a randomized, double-blind, placebo-controlled, parallel-group study in the United States and Europe between August 2013 and August 2014. After a 4-week run-in period, which involved treatment with MFNS, we randomly allocated (1:1) patients to add-on therapy with subcutaneous dupilumab (a 600-mg loading dose followed by weekly doses of $300 \mathrm{mg}$ ) or matched placebo for 16 weeks.

Eligible patients were aged 18 to 65 years with bilateral NP and chronic symptoms of rhinosinusitis despite intranasal corticosteroid treatment for at least 2 months. Patients were required to have a bilateral endoscopic NPS of at least 5 (of a maximum score of 8), with a score of at least 2 for each nostril, and manifest at least 2 of the following symptoms prior to screening: nasal obstruction, reduction or loss of sense of smell, nasal discharge, and facial pain or pressure. Randomization was stratified by medical history of asthma (yes or no) and nasal biopsy (yes or no).

\section{Sinus CT scan assessments}

Sinus CT scans were performed at baseline and Week 16. Images were assessed centrally in a blinded fashion by an independent physician reviewer using 2 validated scoring systems, the 
Table 1. Baseline demographics and clinical characteristics.

\begin{tabular}{|c|c|c|}
\hline & $\begin{array}{l}\text { Placebo } \\
\mathbf{n}=\mathbf{3 0}\end{array}$ & $\begin{array}{l}\text { Dupilumab } \\
\qquad n=30\end{array}$ \\
\hline Age, mean (SD), years & $49.3(9.1)$ & $47.4(9.8)$ \\
\hline Male gender, $\mathrm{n}(\%)$ & $16(53.3)$ & $18(60.0)$ \\
\hline Body mass index, mean (SD) & $26.8(3.9)$ & $28.1(4.2)$ \\
\hline Body mass index $<30, \mathrm{n}(\%)$ & $24(80.0)$ & $22(73.3)$ \\
\hline NP duration, mean (SD), years & $11.5(8.7)$ & $7.6(6.1)$ \\
\hline Comorbid asthma, n (\%) & $19(63.3)$ & $16(53.3)$ \\
\hline Duration of asthma, mean (SD), years & $20.2(17.4)$ & $15.5(12.1)$ \\
\hline $\begin{array}{l}\text { VAS, rhinosinusitis disease severity, mean } \\
\text { (SD), } 0-10^{\mathrm{a}}\end{array}$ & $6.4(2.7)$ & $6.4(2.7)$ \\
\hline SNOT-22 total score, mean (SD), $0-110^{\mathrm{a}}$ & $40.6(19.9)$ & $41.4(18.2)$ \\
\hline $\begin{array}{l}\text { Nasal congestion or obstruction, mean } \\
\text { (SD), } 0-3^{a, b} \\
\text { AM } \\
\text { PM }\end{array}$ & $\begin{array}{l}1.7(0.7) \\
1.6(0.7)\end{array}$ & $\begin{array}{l}1.7(0.7) \\
1.6(0.8)\end{array}$ \\
\hline $\begin{array}{l}\text { Sense of smell loss, mean (SD) }{ }^{a, b} \\
\text { AM } \\
\text { PM }\end{array}$ & $\begin{array}{l}2.8(0.5) \\
2.8(0.5)\end{array}$ & $\begin{array}{l}2.4(0.9) \\
2.4(0.9)\end{array}$ \\
\hline $\begin{array}{l}\text { Bilateral endoscopic nasal polyp score, } \\
\text { mean (SD), } 0-8^{a}\end{array}$ & $5.7(0.9)$ & $5.9(1.0)$ \\
\hline Patients with CT scan assessments, n (\%) & $30(100)$ & $29(96.7)$ \\
\hline LMK CT total score, mean (SD), 0-24 & $18.7(5.5)$ & $18.6(5.0)$ \\
\hline zLMK total score, mean (SD), $0-48^{a}$ & $35.7(10.7)$ & $34.8(8.8)$ \\
\hline $\begin{array}{l}\text { Smell test (UPSIT) score, mean (SD), } \\
0-40^{c}\end{array}$ & $15.6(7.9)$ & $12.8(8.3)$ \\
\hline
\end{tabular}

${ }^{a}$ Higher scores indicate worse status. ${ }^{b}$ Average of the past 7 days before randomization. ${ }^{c}$ Higher scores indicate better status. Abbreviations: AM, morning; CT, computed tomography; LMK, Lund-Mackay; PM, afternoon; SD standard deviation; UPSIT, University of Pennsylvania Smell Identification Test; zLMK, Zinreich modified LMK.

protocol-mandated LMK system, and the more granular zLMK system post hoc ${ }^{(21-26)}$, with a different reviewer for each system. For the LMK CT scoring system, sinuses on both sides were scored using a scale of 0 to $2(0=$ normal; $1=$ partial opacification; and 2 = total opacification). The OMC was scored 0 (normal) or 2 (total) on each side and did not include a score of 1 . The total bilateral LMK score ranges from 0 (complete lucency) to 24 (complete opacity).

The zLMK scoring is based on the percentage opacification of each sinus as follows: $0=0 \% ; 1=1-24 \% ; 2=25-74 \% ; 3=$ $75-99 \% ; 4=100 \%$. The OMC is divided into 4 segments (frontal recess, infundibulum, middle meatus [anterior], and sphenoethmoid recess [posterior]), which are scored bilaterally as 0 or 1 , depending on whether the segment is completely patent $(0)$ or completely obstructed (1). The zLMK system derives a maximum possible score of 24 per side and a total bilateral zLMK score ranging from 0 (complete lack of opacification) to 48 (complete opacity). This system provides increased disease representation and sensitivity to change in partially opacified sinuses.

\section{Correlation analysis: NPS, SNOT-22, UPSIT, daily symptom} scores, VAS severity

Correlation analysis using Pearson's correlation coefficient was conducted at baseline and at Week 16 between total LMK and zLMK scores and the following protocol-defined outcomes, previously reported in Bachert et al. ${ }^{(18)}$ : endoscopic bilateral NPS (0-8), a grading of polyp size and the primary endpoint for this study, with a higher score indicating worse status; patientreported disease severity assessed monthly using visual analogue scale (VAS; $0-10 \mathrm{~cm} ; 0-3 \mathrm{~cm}=$ mild disease, $>3-7 \mathrm{~cm}=$ moderate disease, $>7-10 \mathrm{~cm}=$ severe disease); the sino-nasal outcome test (SNOT-22; total score 0-110), a validated questionnaire used to assess both symptoms and the impact of CRS on HRQoL (27), for which a difference of 8.9 or greater is accepted as the minimally clinically important difference (MCID) ${ }^{(27)}$; SNOT-22 smell/taste item score (score 1-5) derived from the SNOT-22 questionnaire; average of patient reported daily symptom assessments for nasal congestion and/or obstruction, and loss of sense of smell ${ }^{(28)}$, recorded each morning in an electronic diary; and University of Pennsylvania Smell Identification Test (UPSIT) score (0-40), a method to quantitatively assess subjective human olfactory function, which allows the clinician to distinguish patients with a normal sense of smell ("normosmia", indicated by higher UPSIT score) from those with different levels of reduction ("mild, moderate and severe microsmia") or loss ("anosmia", indicated by lower UPSIT score) (29).

\section{Statistical analysis}

Descriptive statistics including frequencies and means were calculated for demographic and baseline characteristics. The change from baseline to Week 16 (end of treatment) for LMK and zLMK was analysed using a mixed-effect model with repeated measures approach. The model included the change from baseline to Week 16 as the response variable, and factors (fixedeffects) for treatment, stratification factors (medical history of asthma and nasal biopsy), visit, treatment-by-visit interaction, NPS baseline value, and baseline-by-visit interaction. An unstructured correlation matrix was used to model the withinpatient errors. Parameters were estimated using the restricted maximum likelihood method with the Newton-Raphson algorithm. No imputation for missing data was performed. Differences in least squares means (LSM) (including corresponding 95\% confidence intervals $[\mathrm{Cl}]$ and $\mathrm{P}$ values) were used to compare dupilumab against placebo. Correlation analysis between total LMK and total zLMK and other endpoints was performed using Pearson's correlation.

\section{Results}

Opacification of sinuses and OMC patency at baseline Baseline demographics and patient characteristics were similar in the placebo and dupilumab groups (Table 1). Baseline bila- 


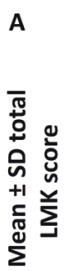

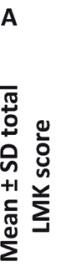

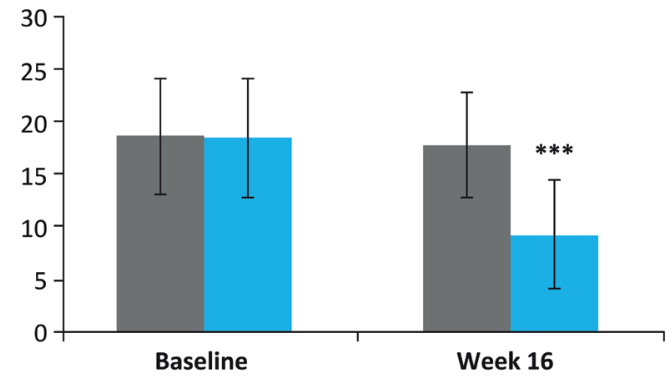

B

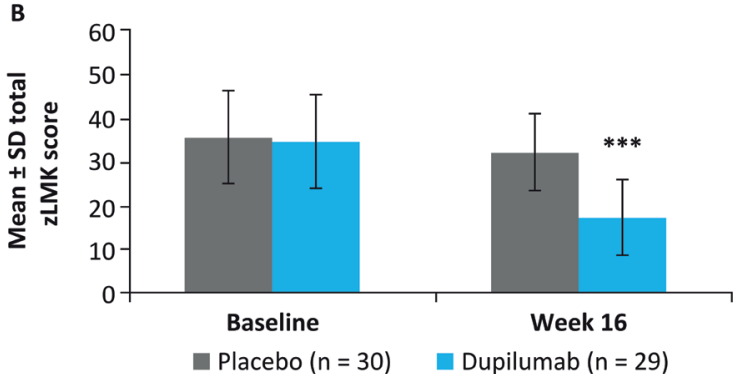

Figure 1. Total mean (SD) LMK (A) and zLMK (B) scores for placebo and dupilumab at baseline and week 16. ${ }^{* *} \mathrm{p}<0.0001$. LMK, LMK, LundMackay; SD, standard deviation; zLMK, Zinreich-modified LMK.
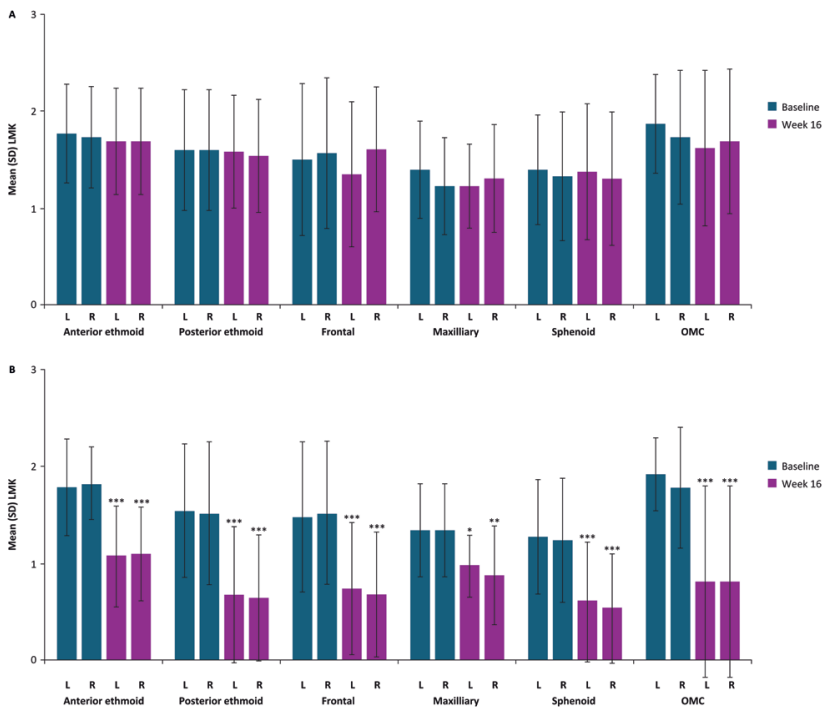

Figure 2. Mean (SD) LMK for placebo (A) and dupilumab (B) at baseline and week 16 by individual sinus, left and right. ${ }^{* *} \mathrm{p}<0.0001$. LMK, LMK, Lund-Mackay; SD, standard deviation; zLMK, Zinreich-modified LMK. teral LMK and zLMK total scores indicated patients had substantial sinus involvement, with mean \pm SD LMK total scores similarly elevated in both the placebo $(18.7 \pm 5.5)$ and dupilumab (18.6 \pm 5.0 ) groups, with the maximum possible LMK score being 24 (Figure $1 \mathrm{~A}$ and Table 1). Mean \pm SD zLMK total scores at baseline were $35.7 \pm 10.7$ and $34.8 \pm 8.8$ for the placebo and dupilumab groups, respectively, with the maximum possible zLMK score being 48 (Figure 1B and Table 1).

At baseline, either a partial or complete opacification was observed across all sinuses using the LMK measure. The anterior ethmoid sinuses presented a high degree of opacification, with mean LMK scores $\geq 1.7$ (range $0.0-2.0$ ) in both the placebo (Figure 2A) and dupilumab (Figure 2B) groups. The posterior ethmoid and frontal sinuses had mean LMK scores $\geq 1.5$ (range $0.0-2.0$ ), while the maxillary and sphenoid sinuses had mean LMK scores $\geq 1.2$ (range $0.0-2.0$ ) for placebo (Figure $2 \mathrm{~A}$ ) and dupilumab (Figure 2B; data for LMK score by sinus, both sides, at baseline are shown in Table S1).

At baseline, the proportion of patients with total opacification (LMK score of 2) ranged from $80 \%$ (for the anterior ethmoid sinus) to $27 \%$ (for the maxillary sinus) in placebo patients (Figure $3 \mathrm{~A}$ ) and from $83 \%$ (for the anterior ethmoid sinus) to $35 \%$ (for the maxillary and sphenoid sinuses) in dupilumab patients (Figure 3B).

At baseline, the OMC was occluded in $\geq 87 \%$ of patients in the placebo group and $\geq 90 \%$ of patients in the dupilumab group (Table S2). Based on the zLMK score at baseline, almost complete obstruction was observed for all 4 segments of the OMC, with scores of 1 in at least $90 \%$ of patients in both groups for the frontal recess and middle meatus segments, in at least $80 \%$ of patients in both groups for the spheno-ethmoidal segment, and in at least $70 \%$ of patients in both groups for the infundibulum segment (the percentages of patients with zLMK scores of 0-1 for OMC left and right at baseline are provided in Table S4). Additional baseline zLMK data, which are provided in Figure 4 and Tables S3 and S4, were similar to the LMK data indicating high degree of opacification, ranging from a mean score of 2.2 (1.4) to 3.3 (0.8) with majority of patients having almost total opacification (75-100\%) of ethmoid and frontal sinuses.

Opacification of sinuses and OMC patency at end of treatment

After 16 weeks of treatment, statistically significant $(p<0.0001)$ improvements with dupilumab vs placebo were observed in total bilateral scores using both standard LMK (LSM difference for dupilumab vs placebo $-8.8 ; 95 \% \mathrm{Cl}-11.1,-6.6)$ and zLMK scoring systems (LSM difference for dupilumab vs placebo -15.4; 95\% Cl-19.3,-11.5) (Figures 1A and 1B). Significant improvements vs placebo were observed for the individual sinuses using the LMK ( $p<0.001$; Figure 2 and Tables S5 and S6) and zLMK CT scoring systems ( $<0.01$; Figure 4 and Tables S7 and S8). At the end of 16 weeks of treatment, the majority of patients treated with dupilumab had a per-sinus LMK score of 0 or 1 (Table S6). Results were similar with the zLMK measure where the majority of patients treated with dupilumab had a per-sinus zLMK score of 0 or 1 ( $<25 \%$ opacification) (Table S8).

At end of treatment, the percentage of patients with complete 
Table 2. Total LMK and zLMK scores by at baseline and week 16.

\begin{tabular}{|c|c|c|}
\hline \multirow[b]{2}{*}{ LMK } & \multicolumn{2}{|c|}{ TOTAL $(\mathbf{N}=60)$} \\
\hline & $\begin{array}{l}\text { Placebo } \\
(n=30)\end{array}$ & $\begin{array}{l}\text { Dupilumab } \\
300 \text { mg qw } \\
(n=30)\end{array}$ \\
\hline $\begin{array}{l}\text { Baseline } \\
\text { Number } \\
\text { Mean (SD) }\end{array}$ & $\begin{array}{c}30 \\
18.73(5.52)\end{array}$ & $\begin{array}{c}29 \\
18.62(5.00)\end{array}$ \\
\hline $\begin{array}{l}\text { Week } 16 \\
\qquad \begin{array}{l}\text { Number } \\
\text { Mean (SD) }\end{array}\end{array}$ & $\begin{array}{c}26 \\
17.92(5.69)\end{array}$ & $\begin{array}{c}30 \\
9.43(5.10)\end{array}$ \\
\hline $\begin{array}{l}\text { Change from baseline } \\
\text { Number } \\
\text { Mean (SD) }\end{array}$ & $\begin{array}{c}26 \\
-0.23(0.95)\end{array}$ & $\begin{array}{c}29 \\
-9.07(0.81)\end{array}$ \\
\hline LS mean difference, $95 \% \mathrm{CI}$ & & $\begin{array}{c}-8.84 \\
(-11.07,-6.61)\end{array}$ \\
\hline$P$ value vs placebo & & $<0.0001$ \\
\hline zLMK & $\begin{array}{l}\text { TOT } \\
\text { Placebo } \\
(n=30)\end{array}$ & $\begin{array}{l}=60) \\
\text { Dupilumab } \\
300 \text { mg qw } \\
\quad(n=30)\end{array}$ \\
\hline $\begin{array}{l}\text { Baseline } \\
\qquad \text { Number } \\
\text { Mean (SD) }\end{array}$ & $\begin{array}{c}30 \\
35.67(10.66)\end{array}$ & $\begin{array}{c}29 \\
34.83(8.82)\end{array}$ \\
\hline $\begin{array}{l}\text { Week } 16 \\
\qquad \begin{array}{l}\text { Number } \\
\text { Mean (SD) }\end{array}\end{array}$ & $\begin{array}{c}26 \\
32.31(10.85)\end{array}$ & $\begin{array}{c}30 \\
17.20(8.78)\end{array}$ \\
\hline $\begin{array}{l}\text { Change from baseline } \\
\text { Number } \\
\text { Mean (SD) }\end{array}$ & $\begin{array}{c}26 \\
-2.23(7.06)\end{array}$ & $\begin{array}{c}29 \\
-17.66(7.71)\end{array}$ \\
\hline LS mean difference, $95 \% \mathrm{Cl}$ & & $\begin{array}{c}-15.37 \\
(-19.28,-11.46)\end{array}$ \\
\hline$P$ value vs placebo & & $<0.0001$ \\
\hline
\end{tabular}

$\mathrm{Cl}$, confidence interval; LMK, Lund-Mackay score; LS, least squares; zLMK, Zinreich-modified Lund-Mackay score.

opacification per sinus in the placebo group remained high (Figure 3A) compared with the dupilumab-treated group (Figure 3B).

Post-treatment obstruction in the OMC measured using the LMK system was $\geq 81 \%$ in the placebo group compared with $\geq 40 \%$ in the dupilumab group (Table S6). Although almost complete obstruction was observed for all 4 segments of the OMC included in the zLMK system at baseline, after 16 weeks of dupilumab treatment at least $40 \%$ of patients had patent frontal recess and middle meatus segments, and more than $60 \%$ of patients had patent infundibulum and spheno-ethmoidal recess segments. In contrast, there were only small improvements in patency across segments in the placebo group (the percentages of patients with zLMK scores of 0-1 for OMC left and right at Week 16 are provided in Table S8).
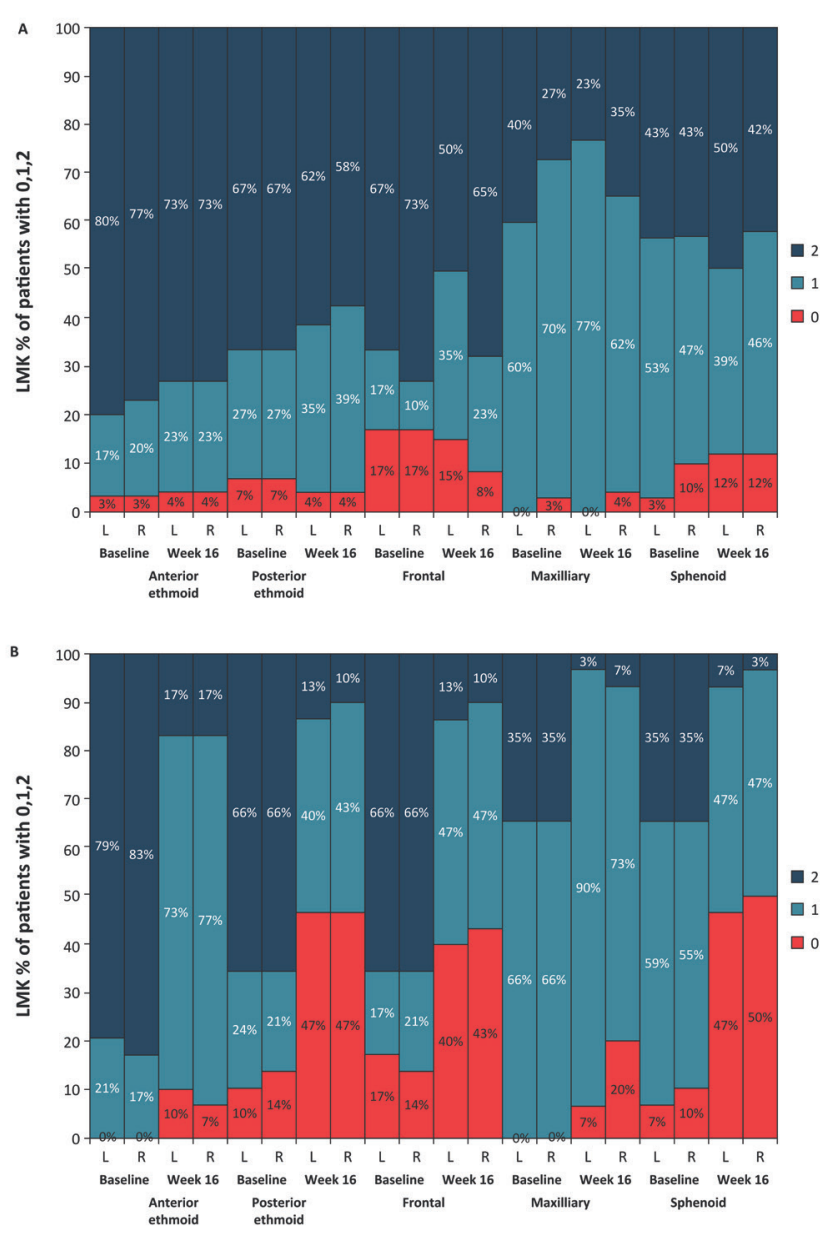

Figure 3. Percentages of patients with LMK score 0, 1, 2 for placebo (A) and dupilumab (B) at baseline and week 16 by sinus, left and right. $L$, left; LMK, Lund-Mackay; R, right.

Correlation between LMK and zLMK Total Scores and other CRSwNP outcomes

For the total patient population (dupilumab and placebo groups pooled, $N=60$ ) at baseline, total LMK score was positively and significantly correlated with bilateral NPS $(r=0.3720, p=$ $0.0037)$, daily assessed loss of sense of smell $(r=0.4579, p=$ $0.0003)$, and the SNOT-22 decreased sense of smell/taste item ( $r$ $=0.4331, p=0.0001$ ), for which high scores indicate more severe disease, and was negatively and significantly correlated with UPSIT, for which a low score indicates more severe disease $(r=$ $-0.6105, p<0.0001)$. These results indicate that opacification measured by LMK correlates with other measures of disease severity. For the patients treated with dupilumab $(n=30)$, at Week 16 total LMK was no longer correlated with any of the assessed outcomes. None of the additional CRSwNP endpoints correlated with LMK at Week 16 in the placebo-treated group (Table S9). At baseline, correlation results for the zLMK measure were similar, while at Week 16 the SNOT-22 decreased sense of smell/ taste item alone remained correlated with $\mathrm{zLMK}(\mathrm{r}=0.3790, \mathrm{p}=$ 


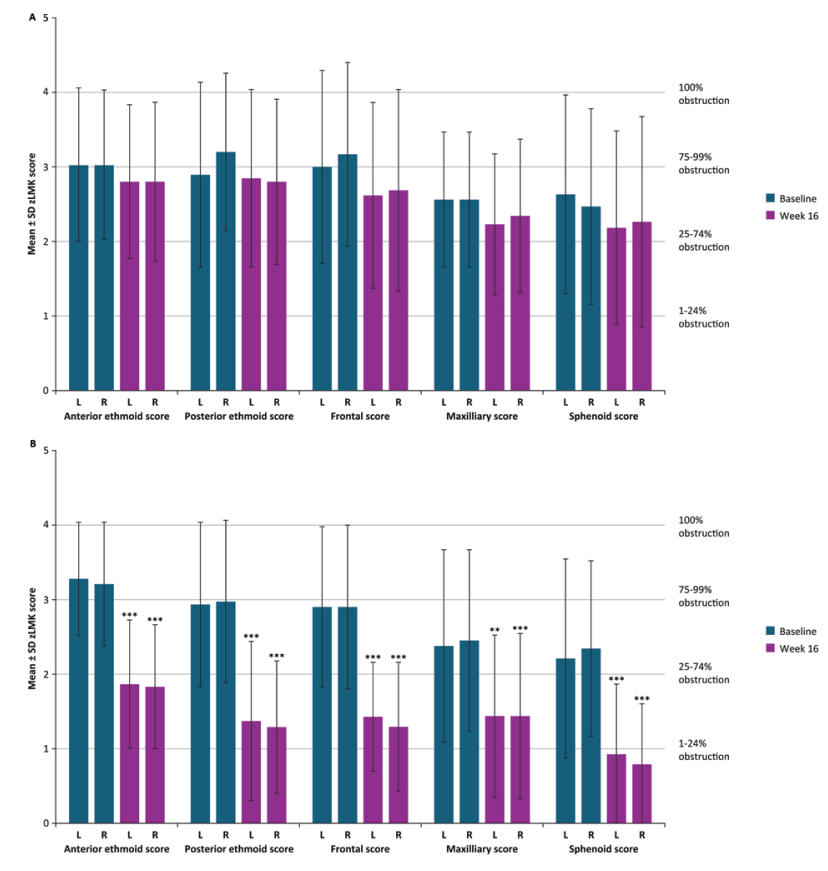

Figure 4. Mean (SD) zLMK for placebo (A) and dupilumab (B) at baseline and week 16 by individual sinus, left and right. L, left; LMK, LundMackay; R, right.

0.0426), in dupilumab-treated patients (Table S10).

\section{Safety}

Safety findings have been reported in detail elsewhere ${ }^{(15)}$. Briefly, 25 of 30 patients in the placebo group and 30 of 30 patients in the dupilumab group reported adverse events. The most common adverse events were nasopharyngitis (placebo, 33\%; dupilumab, 47\%), injection site reactions (placebo, 7\%; dupilumab, 40\%), and headache (placebo, 17\%; dupilumab, 20\%).

\section{Discussion}

In a previous publication, we demonstrated that 16 weeks of treatment with dupilumab in adults with symptomatic CRSwNP refractory to intranasal corticosteroids led to a significant improvement in NPS, LMK, rhinosinusitis severity as assessed by VAS, HRQoL as assessed by SNOT-22, and sense of smell as assessed by UPSIT ${ }^{(15)}$. In this manuscript, we expand the CT scan results to include all sinuses, using the more granular zLMK measuring scale.

In this population of patients with severe CRSwNP (endoscopic NPS $\geq 5$ ), diffuse and substantial opacification of all the sinuses caused by mucosal thickening was observed at baseline, as reflected by high LMK (LMK score of 1-2) and zLMK scores, with most sinuses almost completely opacified. Treatment with dupilumab resulted in reductions in opacification across all sinuses as measured by LMK CT scoring, and in the ZLMK, at Week 16. In this study, we found the mean change from baseline in LMK and zLMK scoring measures to be comparable, in contrast to a previous study reporting that the LMK is less sensitive than the zLMK to small changes in disease severity following intramuscular depot corticosteroid injections ${ }^{(30)}$. Although we would have expected a more distinct gradation with the zLMK scoring system, as was shown in Likness et al. ${ }^{(30)}$, our study included only patients with extensive sinus involvement and polyps at baseline, and this, along with the large treatment effect observed after dupilumab therapy, may account for the lack of difference between the LMK and zLMK scoring systems observed.

The improvements in sinus opacification measures based on both LMK and zLMK were mirrored by improvements in clinical signs (NPS) and symptoms and HRQoL, as reported by Bachert et al. ${ }^{(18)}$. Total sinus opacification as measured by LMK was shown to correlate at baseline with the severity of endoscopic NPS, along with smell test (assessed by UPSIT), the SNOT-22 decreased loss of smell/taste item, and the daily loss of sense of smell, all 3 of which are endpoints related to patient reported ability to smell, suggesting that sinus opacification is related to loss of smell. However, it is worth noting that these correlations were weak to moderate in strength, and that they did not correlate with improvement in LMK score at Week 16 (Table S9). The ability to assess correlations between zLMK and other outcomes based on treatment was limited due to the population size in our study; however, zLMK did positively correlate with decreased loss of smell/taste in the dupilumab group after 16 weeks of treatment.

Previous data regarding correlations between radiological findings and symptoms are mixed ${ }^{(31-34)}$. While data on the relationship between obstruction assessed by CT scan and olfaction are sparse, studies have reported pre-treatment correlations ranging from very weak (Kendall correlation coefficient $=0.112$ ) to strong $(R=-0.71)^{(32,33)}$. Post-dupilumab treatment, the majority of correlations were no longer significant in our study, potentially due to lower $\mathrm{n}$ numbers than those used the pooled baseline group, as mentioned. However, in a previous study office-based CT scans correlated moderately with olfaction post-surgery ${ }^{(34)}$. It has been suggested that reduced olfaction is caused by nasal inflammation, which would be in line with a potential relationship with obstruction ${ }^{(32)}$.

\section{Conclusion}

Dupilumab added to MFNS background therapy significantly decreased opacification across all sinuses after 16 weeks of treatment as measured using the LMK and zLMK scoring systems, while also significantly improving NPS, disease severity, HRQoL, and sense of smell.

\section{Acknowledgements}

Research sponsored by Sanofi and Regeneron Pharmaceuticals, Inc. ClinicalTrials.gov Identifier: NCT01920893. Medical writing/ editorial assistance provided by Adam J. Beech, PhD, of Excerpta 
Medica, funded by Sanofi Genzyme and Regeneron Pharmaceuticals, Inc.

\section{Authorship contribution}

CB, PWH, JM, DLH, PG, RN, NA, VNJ, CF, DZ, HS, GP, NMHG, AK, LPM contributed to the protocol development of the study; $J Z$ contributed to the evaluation of imaging information from the study. All authors acquired data, reviewed and interpreted results and provided direction for manuscript development, and provided critical feedback and final approval for submission

\section{Conflict of interest}

CB: Principal investigator of the study; Sanofi - consultant. J. $J Z$ : Participant in evaluation of imaging information from the study. PWH: No conflicts of interest to disclose. JM: National and international scientific advisory boards (consulting) present or past member; Allakos, ALK-Abelló, FAES, Genentech, GlaxoSmithKline, Hartington Pharmaceuticals, MYLAN/MEDA Pharma, Menarini, Merck Sharp and Dohme, Novartis, SanofiGenzyme, Regeneron Pharmaceuticals, Inc., UCB, Uriach Group - fees for lectures, grants for research projects. DLH: Genentech, Regeneron Pharmaceuticals, Inc., Sanofi - consultancy for use of biologics for chronic rhinosinusitis and nasal polyposis; Merck - grant support for bench research; UpToDate - royalties. PG: Grant support - Sanofi. RN: Genentech, Novartis, Sanofi, ActoBiotics - advisory board; Optinose - speaker's bureau. NA, NMHG: Regeneron Pharmaceuticals, Inc. - employees and shareholders. VNJ: Regeneron Pharmaceuticals, Inc. - former employee and shareholder. CF, DZ, HS, GP, AK, LPM: Sanofi - employees and shareholders.

\section{References}

1. Blackwell DL, Lucas JW, Clarke TC. Summary health statistics for U.S. adults: national health interview survey, 2012. Vital Health Stat 10. 2014; 260: 1-161.

2. Alobid I, Benítez P, Bernal-Sprekelsen M, et al. The impact of asthma and aspirin sensitivity on quality of life of patients with nasal polyposis. Qual Life Res. 2005; 14: 789-793.

3. Alobid I, Benítez P, Valero A, et al. The impact of atopy, sinus opacification, and nasal patency on quality of life in patients with severe nasal polyposis. Otolaryngol Head Neck Surg. 2006; 134: 609-612.

4. Messerklinger W. On the drainage of the normal frontal sinus of man. Acta Otolaryngol. 1967; 63: 176-181.

5. Tomassen P, Vandeplas G, Van Zele T, et al Inflammatory endotypes of chronic rhinosinusitis based on cluster analysis of biomarkers. J Allergy Clin Immunol. 2016; 137: 14491456.e4.

6. Macdonald LE, Karow M, Stevens S, et al. Precise and in situ genetic humanization of $6 \mathrm{Mb}$ of mouse immunoglobulin genes. PNAS. 2014; 111: 5147-5152.

7. Murphy AJ, Macdonald LE, Stevens S, et al. Mice with megabase humanization of their immunoglobulin genes generate antibodies as efficiently as normal mice. PNAS. 2014; 111: 5153-5158.

8. DUPIXENT ${ }^{\oplus}$ (dupilumab). Highlights of prescribing information. US Food and Drug Administration 2019. Available from: https://www.accessdata.fda.gov/drugsatfda_docs/label/2019/761055s012lbl.pdf. Accessed May 2019.

9. Wenzel S, Castro M, Corren J, et al. Dupilumab efficacy and safety in adults with uncontrolled persistent asthma despite use of medium-to-high-dose inhaled corticosteroids plus a long-acting $\beta 2$ agonist: a randomised double-blind placebo-controlled pivotal phase $2 \mathrm{~b}$ doseranging trial. Lancet. 2016; 388: 31-44.
10. Castro M, Corren J, Pavord ID, et al. Dupilumab efficacy and safety in moderateto-severe uncontrolled asthma. $N$ Engl J Med. 2018; 378: 2486-2496.

11. Rabe KF, Nair P, Brusselle G, et al. Efficacy and safety of dupilumab in glucocorticoiddependent severe asthma. N Engl J Med. 2018; 378: 2475-2485

12. DUPIXENT ${ }^{\oplus}$ (dupilumab). Japan PMDA. Available from: http://www.pmda.go.jp/ PmdaSearch/iyakuDetail/ResultDataSetPD F/780069_4490405G1024_1_04. Accessed May 2019.

13. DUPIXENT ${ }^{\oplus}$ (dupilumab). Summary of Product Characteristics. European Medicines Agency 2017. Available from: http://ec.europa.eu/health/ documents/community-register/2019/20190506144541/anx_144541 en.pdf. Accessed May 2019

14. Blauvelt A, de Bruin-Weller M, Gooderham $M$, et al. Long-term management of moderate-to-severe atopic dermatitis with dupilumab and concomitant topical corticosteroids (LIBERTY AD CHRONOS): a 1-year, randomised, double-blinded, placebocontrolled, phase 3 trial. Lancet. 2017; 389: 2287-2303.

15. Simpson EL, Bieber T, Guttman-Yassky E, et al. Two phase 3 trials of dupilumab versus placebo in atopic dermatitis. N Engl J Med. 2016; 375: 2335-2348.

16. Thaçi $D$, Simpson EL, Beck LA, et al. Efficacy and safety of dupilumab in adults with moderate-to-severe atopic dermatitis inadequately controlled by topical treatments: a randomised, placebo-controlled, doseranging phase $2 \mathrm{~b}$ trial. Lancet. 2016; 387: 40-52.

17. Hirano I, Dellon ES, Hamilton JD, et al. Dupilumab efficacy and safety in adult patients with active eosinophilic esophagitis: a randomized double-blind placebocontrolled phase 2 trial. Presented at the 2017 meeting of the World Congress of
Gastroenterology, Orlando, FL, USA.

18. Bachert C, Mannent L, Naclerio RM, et al. Effect of subcutaneous dupilumab on nasal polyp burden in patients with chronic sinusitis and nasal polyposis: a randomized clinical trial. JAMA. 2016; 315: 469-479.

19. Lund VJ, Mackay IS. Staging in rhinosinusitis. Rhinology. 1993; 31: 183-184.

20. Zinreich SJ. Imaging for staging of rhinosinusitis. Ann Otol Rhinol Laryngol Suppl. 2004; 193: 19-23.

21. Lund VJ, MacKay IS. Outcome assessment of endoscopic sinus surgery. J R Soc Med. 1994; 87: 70-72

22. Kennedy DW, Kuhn FA, Hamilos DL, et al. Treatment of chronic rhinosinusitis with high-dose oral terbinafine: a double blind, placebo-controlled study. Laryngoscope. 2005; 115: 1793-1799.

23. Metson R, Gliklich RE, Stankiewicz JA, et al. Comparison of sinus computed tomography staging systems. Otolaryngol Head Neck Surg. 1997; 117: 372-379.

24. Oluwole M, Russell N, Tan L, Gardiner Q, White P. A comparison of computerized tomographic staging systems in chronic sinusitis. Clin Otolaryngol Allied Sci. 1996; 21: 91-95.

25. Okushi T, Nakayama T, Morimoto S, et al. A modified Lund-Mackay system for radiological evaluation of chronic rhinosinusitis. Auris Nasus Larynx. 2013; 40: 548-553.

26. Pallanch J, Yu L, Delone D, et al. 3-D volumetric computed tomographic scoring as an objective outcome measure for chronic rhinosinusitis: clinical correlations and comparison to Lund-Mackay scoring. Int Forum Allergy Rhinol. 2013; 3: 963-972.

27. Hopkins C, Gillett S, Slack R, Lund VJ, Browne JP. Psychometric validity of the 22-item sinonasal outcome test. Clin Otolaryngol. 2009; 34: 447-454.

28. Fokkens W, Lund VJ, Mullol J et al. European Position Paper on Rhinosinusitis and Nasal Polyps Group. EPOS 2012: European posi- 
tion paper on rhinosinusitis and nasal polyps 2012. A summary for otorhinolaryngologists. Rhinol Suppl. 2012; 50: 1-298.

29. Scadding G, Hellings $P$, Alobid I, et al. Diagnostic tools in rhinology EAACI position paper. Clin Transl Allergy. 2011; 1:2.

30. Likness MM, Pallanch JF, Sherris DA, Kita H, Mashtare TL Jr, Ponikau JU. Computed tomography scans as an objective measure of disease severity in chronic rhinosinusitis. Otolaryngol Head Neck Surg. 2014; 150 : 305-311.

31. Rathor A, Bhattacharjee A. Clinicalradiological correlation and role of computed tomography staging in chronic rhinosinusitis. World J Otorhinolaryngol Head Neck Surg. 2017; 3: 169-175.
32. Gupta D, Gulati A, Singh I, Tekur U Endoscopic, radiological, and symptom correlation of olfactory dysfunction in pre- and postsurgical patients of chronic rhinosinusitis. Chem Senses. 2014; 39: 705-710.

33. Kenny TJ, Duncavage J, Bracikowski J, Yildirim A, Murray JJ, Tanner SB. Prospective analysis of sinus symptoms and correlation with paranasal computed tomography scan. Otolaryngol Head Neck Surg. 2001; 125: 40-43

34. Ryan WR, Ramachandra T, Hwang PH. Correlations between symptoms, nasal endoscopy, and in-office computed tomography in post-surgical chronic rhinosinusitis patients. Laryngoscope. 2011; 121: 674-678.
Claus Bachert MD, PhD

Upper Airway Research Laboratory

Department of Otorhinolaryngology

Ghent University Hospital

C. Heymanslaan 10

\section{B-9000 Ghent}

Belgium

Tel: +32 93326880

E-mail:Claus.Bachert@UGent.be 